University of Zurich

Department of Economics

Working Paper Series

ISSN 1664-7041 (print)

ISSN 1664-705X (online)

Working Paper No. 286

\title{
Equilibrium in the Symmetric Hirshleifer Contest: Uniqueness and Characterization
}

Christian Ewerhart and Guang-Zhen Sun

April 2018 


\title{
Equilibrium in the Symmetric Hirshleifer \\ Contest: Uniqueness and Characterization
}

\author{
Christian Ewerhart ${ }^{\dagger}$ \\ Guang-Zhen Sun
}

April 26, 2018

\begin{abstract}
The symmetric two-player Hirshleifer (1989) contest is shown to admit a unique equilibrium. The support of the equilibrium strategy is finite and includes, in particular, the zero expenditure level. We also establish a lower bound for the cardinality of the support and an upper bound for the undissipated rent.
\end{abstract}

Keywords. Contests · Mixed-strategy equilibrium · Rent dissipation · Uniqueness

JEL codes. C72, D72, D74

$\dagger$ (corresponding) Department of Economics, University of Zurich, Schönberggasse 1, CH-8001 Zurich, christian.ewerhart@econ.uzh.ch.

‡) Department of Economics, University of Macau, gzsun@umac.mo. 


\section{Introduction}

Mixed equilibria in contests of the generalized Tullock form, for which winning probabilities depend on the ratio of resources expended, have recently received much attention from theorists (Baye et al., 1994; Alcade and Dahm, 2010; Ewerhart, 2015, 2017a, 2017b; Feng and Lu, 2017). There is another appealing class of contests, however, where the winning probabilities depend instead on the difference of resources expended (Hirshleifer, 1989; Skaperdas, 1996; Baik, 1998; Che and Gale, 2000). In particular, Hirshleifer's framework has its merits for the analysis of military combat (Dupuy, 1987; Hirshleifer, 2000). Notwithstanding, the nature of mixed equilibria in that model has remained poorly understood.

In this paper, we prove uniqueness of the equilibrium in the symmetric two-player Hirshleifer contest, and offer a characterization of the mixed equilibrium. It is shown that the support of the symmetric equilibrium strategy is finite and includes the origin. Moreover, the cardinality of the support grows over any finite bound as the decisiveness parameter goes to infinity. Further, we show that the undissipated rent converges to zero as the decisiveness parameter goes to infinity, and that ex-post overdissipation may occur. We conclude by extending the uniqueness result to a larger class of contests.

The uniqueness result is stated in Section 2, and proven in Section 3. Section 4 characterizes the equilibrium. Rent dissipation is dealt with in Section 5. Section 6 discusses ex-post overdissipation. Alternative contest technologies are considered in Section 7. 


\section{Statement of the uniqueness result}

The Hirshleifer contest is specified as follows. Each of two players $i \in\{1,2\}$ expends resources $x_{i} \geq 0$ in an attempt to win a prize of normalized value one. Player $i$ 's payoff is given as

$$
\begin{aligned}
\Pi_{i}\left(x_{i}, x_{j}\right) & =\frac{\exp \left(\alpha x_{i}\right)}{\exp \left(\alpha x_{i}\right)+\exp \left(\alpha x_{j}\right)}-x_{i} \\
& =\frac{1}{1+\exp \left(\alpha\left(x_{j}-x_{i}\right)\right)}-x_{i},
\end{aligned}
$$

where $j \in\{1,2\}$ with $j \neq i$, and $\alpha>0$ measures the decisiveness of the difference-form contest. In particular, for $\alpha \rightarrow \infty$, payoffs converge against those of the all-pay auction.

Any bid exceeding one is strictly dominated. We therefore define a mixed strategy for player $i$ as a probability measure $\mu_{i}$ on the Borel subsets of $[0,1]$. The set of mixed strategies for player $i$ will be denoted by $M$, where pure strategies $x_{i} \in[0,1]$ are interpreted as Dirac measures, as usual. Each player $i$ 's expected payoff is well-defined for any $\left(\mu_{i}, \mu_{j}\right) \in M \times M$, and will, with some abuse of notation, be denoted by $\Pi_{i}\left(\mu_{i}, \mu_{j}\right)$. An equilibrium is a pair $\mu^{*}=\left(\mu_{1}^{*}, \mu_{2}^{*}\right) \in M \times M$ such that $\Pi_{i}\left(\mu_{i}^{*}, \mu_{j}^{*}\right) \geq \Pi_{i}\left(\mu_{i}, \mu_{j}^{*}\right)$ for any $i, j \in\{1,2\}$ with $j \neq i$, and for any $\mu_{i} \in M$.

Proposition 1. For any $\alpha>0$, the Hirshleifer contest with parameter $\alpha$ has a unique equilibrium.

\section{Proof of Proposition 1}

Equilibrium existence is known (cf. Hirshleifer, 1989, fn. 12). The proof of uniqueness starts from the following observation.

Lemma 1. Let $\mu=\left(\mu_{1}, \mu_{2}\right) \in M \times M$. Then, for any $i, j \in\{1,2\}$ with $j \neq i$, the set of maximizers $X_{i}(\mu)=\arg \max _{\widetilde{x}_{i} \in[0,1]} \Pi_{i}\left(\widetilde{x}_{i}, \mu_{j}\right)$ is finite. 
Proof. The proof is a straightforward adaption of Ewerhart (2015, Th. 3.2 , and therefore omitted. ${ }^{1}$

Next, we show the following.

Lemma 2. The set $X^{\alpha}=\bigcap_{\mu^{*} \text { equilibrium }} X_{1}\left(\mu^{*}\right)$ is nonempty, and contains the support of any equilibrium strategy (for both players).

Proof. Take an equilibrium $\mu^{*}=\left(\mu_{1}^{*}, \mu_{2}^{*}\right)$. Clearly, the support of $\mu_{1}^{*}$ is a subset of $X_{1}\left(\mu^{*}\right)$. Let $\mu^{* *}=\left(\mu_{1}^{* *}, \mu_{2}^{* *}\right)$ be an arbitrary equilibrium. Then, since equilibria in two-player contests are interchangeable (Ewerhart, 2017b, Appendix), $\left(\mu_{1}^{*}, \mu_{2}^{* *}\right)$ is an equilibrium. Therefore, the support of $\mu_{1}^{*}$ is a subset of $X_{1}\left(\mu_{1}^{*}, \mu_{2}^{* *}\right)$. But $X_{1}\left(\mu_{1}^{*}, \mu_{2}^{* *}\right)=X_{1}\left(\mu^{* *}\right)$. Hence, the support of $\mu_{1}^{*}$ is contained in $X_{1}\left(\mu^{* *}\right)$ for any equilibrium $\mu^{* *}$. In particular, $X^{\alpha} \neq \varnothing$. The second claim follows by symmetry.

Denote by $K=\left|X^{\alpha}\right|$ the number of elements of $X^{\alpha}$. Thus, $X^{\alpha}=\left\{z_{1}, \ldots, z_{K}\right\}$, where $z_{1}>z_{2}>\ldots>z_{K}$. Suppose first that $K=1$. Then, the equilibrium is obviously unique. Suppose next that $K \geq 2$. Fix some equilibrium $\mu^{*}=\left(\mu_{1}^{*}, \mu_{2}^{*}\right)$, and let $p_{j}^{m}=\mu_{i}^{*}\left(\left\{z_{m}\right\}\right) \geq 0$ denote the weight assigned by $\mu_{j}^{*}$ to $z_{m}$, for $j \in\{1,2\}$ and $m \in\{1, \ldots, K\}$. We know that $z_{1}, \ldots, z_{K}$ all deliver the equilibrium payoff $\Pi_{i}^{*}$ against $\mu_{j}^{*}$, i.e.,

$$
\Pi_{i}^{*}=\left(\sum_{m=1}^{K} p_{j}^{m} \frac{\exp \left(\alpha z_{k}\right)}{\exp \left(\alpha z_{k}\right)+\exp \left(\alpha z_{m}\right)}\right)-z_{k} \quad(k=1, \ldots, K ; j \neq i) .
$$

Thus, there are $K$ equations to identify $(K+1)$ unknowns $p_{j}^{1}, \ldots, p_{j}^{K}$ and $\Pi_{i}^{*}$. Notably, adding the relationship $\sum_{m=1}^{K} p_{j}^{m}=1$ does not help in general. Instead, we focus on the largest element of the support of player $i$ 's equilibrium strategy. ${ }^{2}$ Since $K \geq 2$, we know that $z_{1}$ is an interior maximum.

\footnotetext{
${ }^{1}$ If attention is restricted to strategies that are absolutely continuous with respect to the Lebesgue measure, the use of complex-analytic methods may be circumvented (Sun, 2017).

${ }^{2}$ The first-named author would like to thank Larry Samuelson for this suggestion.
} 
Hence, the first-order condition implies

$$
\sum_{m=1}^{K} p_{j}^{m} \frac{\alpha \exp \left(\alpha z_{1}\right) \exp \left(\alpha z_{m}\right)}{\left(\exp \left(\alpha z_{1}\right)+\exp \left(\alpha z_{m}\right)\right)^{2}}=1 .
$$

Combining these $(K+1)$ equations yields

$$
\left(\begin{array}{cccc}
\frac{\exp \left(\alpha z_{1}\right)}{\exp \left(\alpha z_{1}\right)+\exp \left(\alpha z_{1}\right)} & \cdots & \frac{\exp \left(\alpha z_{1}\right)}{\exp \left(\alpha z_{1}\right)+\exp \left(\alpha z_{K}\right)} & 1 \\
\vdots & \ddots & \vdots & \vdots \\
\frac{\exp \left(\alpha z_{K}\right)}{\exp \left(\alpha z_{K}\right)+\exp \left(\alpha z_{1}\right)} & \cdots & \frac{\exp \left(\alpha z_{K}\right)}{\exp \left(\alpha z_{K}\right)+\exp \left(\alpha z_{K}\right)} & 1 \\
\frac{\alpha \exp \left(\alpha z_{1}\right) \exp \left(\alpha z_{1}\right)}{\left(\exp \left(\alpha z_{1}\right)+\exp \left(\alpha z_{1}\right)\right)^{2}} & \cdots & \frac{\alpha \exp \left(\alpha z_{1} \exp \left(\alpha z_{K}\right)\right.}{\left(\exp \left(\alpha z_{1}\right)+\exp \left(\alpha z_{K}\right)\right)^{2}} & 0
\end{array}\right)\left(\begin{array}{c}
p_{j}^{1} \\
\vdots \\
p_{j}^{K} \\
-\Pi_{i}^{*}
\end{array}\right)=\left(\begin{array}{c}
z_{1} \\
\vdots \\
z_{K} \\
1
\end{array}\right) \text {. }
$$

It turns out that (5) has at most one solution.

Lemma 3. The square matrix on the left-hand side of (5) is invertible.

Proof. Let $e_{k}=\exp \left(\alpha z_{k}\right)$ for $k=1, \ldots, K$, and

$$
A_{1}=\left(\begin{array}{cccc}
\frac{e_{1}}{e_{1}+e_{1}} & \cdots & \frac{e_{1}}{e_{1}+e_{K}} & 1 \\
\frac{e_{2}}{e_{2}+e_{1}} & \cdots & \frac{e_{2}}{e_{2}+e_{K}} & 1 \\
\vdots & \ddots & \vdots & \vdots \\
\frac{e_{K}}{e_{K}+e_{1}} & \cdots & \frac{e_{K}}{e_{K}+e_{K}} & 1 \\
\frac{\alpha e_{1} e_{1}}{\left(e_{1}+e_{1}\right)^{2}} & \cdots & \frac{\alpha e_{1} e_{K}}{\left(e_{1}+e_{K}\right)^{2}} & 0
\end{array}\right) .
$$

Subtracting row $k=1$ from row $k$, for $k=2, \ldots, K$, yields $\operatorname{det} A_{1}=\operatorname{det} A_{2}$, where

$$
A_{2}=\left(\begin{array}{cccc}
\frac{e_{1}}{e_{1}+e_{1}} & \cdots & \frac{e_{1}}{e_{1}+e_{K}} & 1 \\
\frac{\left(e_{2}-e_{1}\right) e_{1}}{\left(e_{2}+e_{1}\right)\left(e_{1}+e_{1}\right)} & \cdots & \frac{\left(e_{2}-e_{1}\right) e_{K}}{\left(e_{2}+e_{K}\right)\left(e_{1}+e_{K}\right)} & 0 \\
\vdots & \ddots & \vdots & \vdots \\
\frac{\left(e_{K}-e_{1}\right) e_{1}}{\left(e_{K}+e_{1}\right)\left(e_{1}+e_{1}\right)} & \cdots & \frac{\left(e_{K}-e_{1}\right) e_{K}}{\left(e_{K}+e_{K}\right)\left(e_{1}+e_{K}\right)} & 0 \\
\frac{\alpha e_{1} e_{1}}{\left(e_{1}+e_{1}\right)^{2}} & \cdots & \frac{\alpha e_{1} e_{K}}{\left(e_{1}+e_{K}\right)^{2}} & 0
\end{array}\right) .
$$

Next, we extract the factor $e_{m} /\left(e_{1}+e_{m}\right)>0$ from column $m$, for $m=$ $1, \ldots, K$, and the factor $\left(e_{k}-e_{1}\right)>0$ from row $k$, for $k=2, \ldots, K$. Further, we extract the factor $\alpha e_{1}>0$ from the last row. This yields

$$
\operatorname{det} A_{2}=\left(\prod_{1 \leq m \leq K} \frac{e_{m}}{e_{1}+e_{m}}\right) \cdot\left(\prod_{2 \leq k \leq K}\left(e_{k}-e_{1}\right)\right) \cdot \alpha e_{1} \cdot \operatorname{det} A_{3},
$$


where

$$
A_{3}=\left(\begin{array}{ccccc}
\frac{e_{1}}{e_{1}} & \frac{e_{1}}{e_{2}} & \cdots & \frac{e_{1}}{e_{K}} & 1 \\
\frac{1}{e_{2}+e_{1}} & \frac{1}{e_{2}+e_{2}} & \cdots & \frac{1}{e_{2}+e_{K}} & 0 \\
\vdots & \vdots & \ddots & \vdots & \vdots \\
\frac{1}{e_{K}+e_{1}} & \frac{1}{e_{K}+e_{2}} & \cdots & \frac{1}{e_{K}+e_{K}} & 0 \\
\frac{1}{e_{1}+e_{1}} & \frac{1}{e_{1}+e_{2}} & \cdots & \frac{1}{e_{1}+e_{K}} & 0
\end{array}\right) .
$$

Finally, we exchange row 1 and row $K+1$. Therefore, $\operatorname{det} A_{3}=-\operatorname{det} A_{4}$, where $A_{4}=\left\{\frac{1}{e_{k}+e_{m}}\right\}_{k=1, \ldots, K ; m=1, \ldots, K}$ is a Cauchy matrix (e.g., Krattenthaler, 2001) with

$$
\operatorname{det} A_{4}=\frac{\prod_{1 \leq k<m \leq K}\left(e_{k}-e_{m}\right)^{2}}{\prod_{1 \leq k \leq K, 1 \leq m \leq K}\left(e_{k}+e_{m}\right)} \neq 0 .
$$

This proves the lemma.

Recall that the support of any equilibrium is contained in $X^{\alpha}=\left\{z_{1}, \ldots, z_{K}\right\}$. Hence, with probabilities $p_{j}^{1}, \ldots, p_{j}^{K}$ being unique, there can indeed be at most one equilibrium.

\section{Characterization ${ }^{3}$}

Since the Hirshleifer contest with parameter $\alpha>0$ admits only one equilibrium, the two players necessarily use the same equilibrium strategy $\mu_{\alpha} \in M$. The following result characterizes $\mu_{\alpha}$.

Proposition 2. Let $\alpha>0$. Then, the following properties hold:

(i) $\mu_{\alpha}$ has finite support $\left\{y_{1}, \ldots, y_{L}\right\}$, where $y_{1}>\ldots>y_{L}$, with $L \geq \frac{\alpha}{4}$.

(ii) $\mu_{\alpha}$ has a mass point at the zero bid, i.e., $y_{L}=0$.

(iii) there is two-sided peace (i.e., $L=1$ ) if and only if $\alpha \leq 4$.

Proof. (i) By Lemma 1, the support of $\mu_{\alpha}$ is finite. Denote by $q_{m}=$ $\mu_{\alpha}\left(\left\{y_{m}\right\}\right)>0$ the probability assigned to $y_{m}$, for $m \in\{1, \ldots, L\}$. From the

\footnotetext{
${ }^{3}$ This section and the next supersede the corresponding parts of earlier work by the authors (Ewerhart, 2014; Sun, 2017).
} 
KKT conditions,

$$
\frac{q_{l}}{4}+\sum_{\substack{m=1 \\ m \neq l}}^{L} \frac{q_{m} \exp \left(\alpha y_{m}\right) \exp \left(\alpha y_{l}\right)}{\left(\exp \left(\alpha y_{l}\right)+\exp \left(\alpha y_{m}\right)\right)^{2}} \leq \frac{1}{\alpha} \quad(l=1, \ldots, L),
$$

with equality for $l=1, \ldots, L-1$, so that $q_{l} \leq \frac{4}{\alpha}$ for any $l=1, \ldots, L$. Therefore, $L \geq \frac{\alpha}{4}$. (ii) For $L=1$, the claim is due to Hirshleifer (1989). Suppose next that $L \geq 2$. Then, player $i$ 's expected payoff against $\mu_{\alpha}$ at the smallest mass point $y_{L}$ satisfies

$$
\begin{aligned}
& \frac{\partial^{2} \Pi_{i}\left(y_{L}, \mu_{\alpha}\right)}{\partial x_{i}^{2}} \\
= & \sum_{m=1}^{L-1} \frac{q_{m} \alpha^{2}\left(\exp \left(\alpha y_{m}\right)-\exp \left(\alpha y_{L}\right)\right) \exp \left(\alpha y_{L}\right) \exp \left(\alpha y_{m}\right)}{\left(\exp \left(\alpha y_{L}\right)+\exp \left(\alpha y_{m}\right)\right)^{3}} \\
> & 0,
\end{aligned}
$$

which shows that $y_{L}$ cannot be an interior maximum. Hence, $y_{L}=0$. (iii) Hirshleifer (1989) has shown that two-sided peace is an equilibrium for $\alpha \leq 4$. For $\alpha>4$, however, part (i) implies $L \geq 2$.

Example $(L=2)$. Consider an equilibrium strategy $\mu_{\alpha}$ that places probability $q_{1}>0$ on $y_{1}>0$, and probability $1-q_{1}>0$ on $y_{2}=0$. Then,

$$
\begin{aligned}
& y_{1}=\frac{1}{2}-\frac{1}{1+\exp \left(\alpha y_{1}\right)}, \\
& q_{1}=1-\frac{\alpha-4}{4 \alpha y_{1}^{2}} .
\end{aligned}
$$

This equilibrium exists numerically for $\alpha \in(4,6.79) .{ }^{4}$

\footnotetext{
${ }^{4}$ The implicit value for $y_{1}$ may be characterized alternatively in terms of the $r$-Lambert function (Mesö and Baricz, 2017).
} 


\section{Rent dissipation}

Rent dissipation is always incomplete in the Hirshleifer contest. ${ }^{5}$ For $\alpha \rightarrow$ $\infty$, however, the equilibrium payoff $\Pi^{*}$ goes to zero, as the following result shows.

Proposition 3. $\Pi^{*} \leq \frac{2}{\alpha}$.

\section{Proof.}

$$
\begin{aligned}
\Pi^{*} & =\Pi_{i}\left(0, \mu_{\alpha}\right) \\
& =\sum_{l=1}^{L} \frac{q_{l}}{1+\exp \left(\alpha y_{l}\right)} \\
& \leq 2 \sum_{l=1}^{L} \frac{q_{l}}{1+\exp \left(\alpha y_{l}\right)} \cdot \frac{\exp \left(\alpha y_{l}\right)}{1+\exp \left(\alpha y_{l}\right)} \\
& =\frac{2}{\alpha}\left(\frac{\partial \Pi_{i}\left(0, \mu_{\alpha}\right)}{\partial x_{i}}+1\right) .
\end{aligned}
$$

Since $\partial \Pi_{i}\left(0, \mu_{\alpha}\right) / \partial x_{i} \leq 0$, the claim follows.

Figure 1 outlines the equilibrium payoff $\Pi^{*}$ and its upper bound as a function of $\alpha$. Note that $\Pi^{*}$, contrary to intuition, is not globally declining. For example, if $\alpha=6.1(<6.6)$, the equilibrium is given by $y_{1}=0.4337(0.4517)$, $q_{1}=0.5425(0.5173)$, and $\Pi^{*}=0.2646(<0.2662)$. Thus, the increase in $y_{1}$ is more than compensated by a decline in $q_{1}$.

\footnotetext{
${ }^{5}$ This fact contrasts, of course, with the complete rent dissipation pervasive in sufficiently decisive contests of the ratio form (Baye et al., 1994; Alcade and Dahm, 2010; Ewerhart, 2015, 2017a).
} 


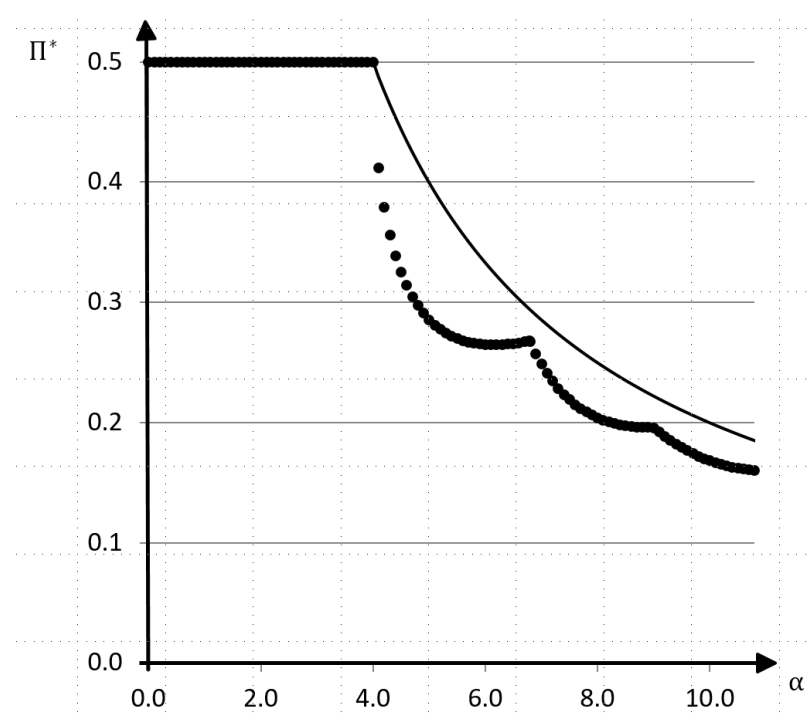

Figure 1. Undissipated rent as a function of $\alpha$.

\section{Ex-post overdissipation}

Although rent dissipation is less severe than in the Tullock case, the Hirshleifer contest may nevertheless feature ex-post overdissipation, i.e., the sum of realized bids may exceed the value of the prize with positive probability (cf. Baye et al., 1999).

Proposition 4. $y_{1} \geq \frac{3}{4}-\frac{10}{3 \alpha}$.

Proof. Recall that $y_{1}>\ldots>y_{L}$. Let $L^{*}$ be the largest $l \in\{1, \ldots, L\}$ such that

$$
\frac{\exp \left(\alpha y_{1}\right)}{\exp \left(\alpha y_{1}\right)+\exp \left(\alpha y_{l}\right)}<\frac{3}{4}
$$

and let

$$
Q=\sum_{l=1}^{L^{*}} q_{l} .
$$

Then, by Proposition 3,

$$
\frac{2}{\alpha} \geq \Pi^{*} \geq \frac{3}{4}(1-Q)+\frac{1}{2} Q-y_{1}
$$


Therefore,

$$
Q \geq 3-4 y_{1}-\frac{8}{\alpha} .
$$

But, from the first-order condition at $y_{1}$,

$$
1 \geq \alpha \sum_{l=1}^{L^{*}} q_{l} \frac{\exp \left(\alpha y_{1}\right) \exp \left(\alpha y_{l}\right)}{\left(\exp \left(\alpha y_{1}\right)+\exp \left(\alpha y_{l}\right)\right)^{2}} \geq \alpha \cdot Q \cdot \frac{3}{4} \cdot \frac{1}{4} .
$$

Using (23) in (24) yields the claim.

Proposition 4 implies that ex-post overdissipation occurs for any sufficiently large $\alpha$. Using numerical analysis, we verified that $y_{1}>0.5$ holds for $\alpha>7.2$.

\section{Alternative contest technologies}

Consider a contest technology of the form

$$
\Pi_{i}^{h}\left(x_{i}, x_{j}\right)=\frac{h\left(x_{i}\right)}{h\left(x_{i}\right)+h\left(x_{j}\right)}-x_{i},
$$

where $h>0$ is a positive impact function (as in Neary, 1997). Provided that $h$ admits, in addition, a real-analytic extension to $(-\varepsilon, \infty)$, for some $\varepsilon>0$, the uniqueness argument goes through. In that case, however, the equilibrium need no longer possess a mass point at the origin. ${ }^{6}$

\section{References}

Alcalde, J., Dahm, M. (2010), Rent seeking and rent dissipation: A neutrality result, Journal of Public Economics 94, 1-7.

Amegashie, J.A. (2006), A contest success function with a tractable noise parameter, Public Choice 126, 135-144.

Baik, K.H. (1998), Difference-form contest success functions and effort levels in contests, European Journal of Political Economy 14, 685-701.

\footnotetext{
${ }^{6}$ E.g., there is no mass point at zero for $h\left(x_{i}\right)=\eta+x_{i}$ if $\eta<1 / 4$ (Amegashie, 2006).
} 
Baye, M.R., Kovenock, D., De Vries, C.G. (1994), The solution to the Tullock rent-seeking game when $R>2$ : Mixed-strategy equilibria and mean dissipation rates, Public Choice 81, 363-380.

— (1999), The incidence of overdissipation in rent-seeking contests, Public Choice 99, 439-454.

Che, Y.K., Gale, I. (2000), Difference-form contests and the robustness of all-pay auctions, Games and Economic Behavior 30, 22-43.

Dupuy, T.N. (1987), Understanding War: History and Theory of Combat, Nova Publications, New York.

Ewerhart, C. (2014), Elastic contests and the robustness of the all-pay auction, ECON Working Paper No. 155, University of Zurich.

- (2015), Mixed equilibria in Tullock contests, Economic Theory 60, 59-71.

- (2017a), Contests with small noise and the robustness of the all-pay auction, Games and Economic Behavior 105, 195-211.

— (2017b), Revenue ranking of optimally biased contests: The case of two players, Economics Letters 157, 167-170.

Feng, X., Lu, J. (2017), Uniqueness of equilibrium in two-player asymmetric Tullock contests with intermediate discriminatory power, Economics Letters 159, 61-64.

Hirshleifer, J. (1989), Conflicts and rent-seeking success functions: Ratio vs difference models of relative success, Public Choice 63, 101-112.

- (2000), The macrotechnology of conflict, Journal of Conflict Resolution 44, 773-792.

Krattenthaler, C. (2001), Advanced determinant calculus, pp. 349-426 in: The Andrews Festschrift, Springer, Berlin. 
Mezö, I., Baricz, Á. (2017), On the generalization of the Lambert $W$ function, Transactions of the American Mathematical Society 369, 7917-7934.

Neary, H.M. (1997), Equilibrium structure in an economic model of conflict, Economic Inquiry 35, 480-494.

Skaperdas, S. (1996), Contest success functions, Economic Theory 7, 283290.

Sun, G.-Z. (2017), Characterizing the symmetric mixed-strategy equilibrium in rent-seeking contests: A real analysis approach, University of Macau. 